\title{
Algorithm Development in Computational Materials Science
}

\author{
JONATHAN A. ZIMMERMAN,,${ }^{1,6}$ ADRIAN S. SABAU, ${ }^{2}$ MOHSEN ASLE \\ ZAEEM, ${ }^{3}$ MARK A. TSCHOPP, ${ }^{4}$ and DOUGLAS E. SPEAROT ${ }^{5}$ \\ 1.--Mechanics of Materials Department, Sandia National Laboratories, Livermore, CA 94550, \\ USA. 2.-Materials Science \& Technology Division, Oak Ridge National Laboratory, Oak Ridge, \\ TN 37831, USA. 3.-Materials Science \& Engineering Department, Missouri University of Science \\ and Technology, Rolla, MO 65409, USA. 4.-Materials and Manufacturing Science Division, U.S. \\ Army Research Laboratory, Adelphi, MD 20783, USA. 5.-Department of Mechanical Engineer- \\ ing, University of Arkansas, Fayetteville, AR 72701, USA. 6.-e-mail: jzimmer@sandia.gov
}

Computational modeling and simulation are among the areas of research, development, and application that have had the most impact on the field of materials science and engineering. This fact was recently underscored when the 2013 Nobel Prize in Chemistry was awarded to Martin Karplus, Michael Levitt, and Arieh Warshel for developing multiscale models for complex chemical systems. This accomplishment-made initially in the mid1970 s-affirms the role that numerical simulation has on elucidating the physical behavior of materials and development of new materials. It also highlights that in an arena where computers are tasked with doing the 'heavy lifting' of mathematical calculations, innovation is still needed to leverage their power to attain true scientific achievement. For instance, the work by Karplus, Levitt, and Warshel is notable not only for developing and implementing a computer program to compute $\pi$-electron and vibration spectra of planar molecules but also for coupling classic and quantum approaches to enable a computationally efficient approach that made modeling the phenomenon of protein folding a feasible endeavor.

Today, the use of computation in materials research is far more pervasive than it was in the $1970 \mathrm{~s}$, and while the resources available in the 21st century far surpass those available to Karplus, Levitt, and Warshel in the 1970s, the need for innovative techniques and algorithms is no less critical. Just as computational power has grown dramatically since those early days, so too have increased the number and complexity of methods by which modeling, simulation, and analysis is done. These methods exist over a gamut of length and time scales, and they include techniques such as density functional theory, interatomic potential (i.e., force field)-based molecular dynamics, the Monte Carlo method, discrete dislocation dynamics, cellular automata method, the phase-field method, finite-element analysis, and many more. While the foundations of all of these approaches already exist, the development of solution algorithms is ongoing to enable the treatment of increasingly complex systems and materials over longer spans of simulated time in an acceptable amount of computational time. The complexity of multiscale and multiphysics models is the key issue, with the goal of improving the representation of the relevant physical and chemical processes being paramount. Strategies to achieve this complexity vary from extending existing methods into foreign regimes of length-timeenergy-phase space, to the coupling of multiple methods, each firmly rooted in its own regime, to the design of new methods that explore this landscape in unique and novel ways.

Developing, implementing, and executing simulation methods is only half of the challenges faced by the modern computational scientist. Data analysis and characterization of computer simulation results that are relevant to each computational approach require an equal effort developing techniques and tools able to sift through gigabytes to terabytes of data and delivering insights on the simulated structures and processes. For example, to study defects in crystalline media during atomistic simulation, common neighbor analysis, ${ }^{1}$ centrosymmetry parameter, ${ }^{2}$ and the dislocation extraction algorithm $^{3}$ represent a few notable developments that have enabled characterizing material structure within simulated configurations. These insights necessitate not only the efficient calculation of experimentally measurable characteristics of material systems but also the theoretical development of new metrics that offer new understanding not provided by experiments.

The need for innovation in algorithm development is not restricted to activities of the computational theoretician. The hardware and techniques available to the experimental materials scientist have also evolved over time, necessitating algorithms that expand the frontiers of data acquisition and analysis. Approaches for extracting useful information from large sets of data-known as data 
mining - have been pursued as they present unique challenges to the computational materials scientist. One such example is the work by Torquato and colleagues, ${ }^{4-6}$ who have investigated and developed statistical metrics capable of representing and reconstructing the complex microstructures of heterogeneous and amorphous materials. Such advancements potentially have positive repercussions on modeling and simulation too, as they can be used in the analysis of numerical results. Furthermore, all of these areas are impacted by innovation in algorithms that can leverage specific computer architectures for revolutionary improvements in simulation analysis time, power, and capability.

The topic of algorithm development in computational materials science and engineering was the focus of a symposium held at the recent 2014 TMS Annual Meeting \& Exhibition. Technical presentations covered a variety of topics ranging from new models, to predicting grain structure formation during solidification, to topological characterization of three-dimensional microstructures with diffuse interfaces, to mapping the stochastic response of nanostructures. The themes covered by this symposium included the development of algorithms for quantum calculations and atomistic simulation, materials chemistry and phase transformations, mesoscale modeling, bridging of simulation across several length and time scales, macroscopic plastic deformation, and integrating experimental techniques.

In this issue of JOM, we present reviews and recent contributions of algorithm development that have advanced computational materials science and engineering, thus providing a narrow but meaningful glimpse of the work presented at the aforementioned symposium. Alexander Stukowski briefly discusses computational analysis methods used for atomistic modeling of crystals, including recent developments that aid in the identifying defect structures in these materials. Shawn P. Coleman, Mehrdad M. Sichani, and Douglas E. Spearot discuss an exciting characterization algorithm to produce virtual $\mathrm{x}$-ray and electron diffraction "data" from atomistic simulation output. Jason F. Panzarino and Timothy J. Rupert discuss a new algorithm for identifying crystallites, and characterizing their orientation and texture in atomistic simulations. All three contributions offer novel methods to extricate vital materials characterization information from complex molecular simulation results for cases where simple visualization techniques would be limiting.

Connecting the atomic to larger length scales, Ebrahim Asadi, Mohsen Asle Zaeem, and Michael I. Baskes discuss an approach whereby molecular dynamics simulation results are used to parameterize the recently developed phase-field crystal approach. Ricardo A. Lebensohn and Reeju Pokharel also reaches into the microstructural level of materials modeling by discussing a novel formulation and algorithms for fast Fourier transform (FFT)-based micromechanical modeling of polycrystalline materials. Finally, Dongsheng Li discusses a review of structure representation and reconstruction that have applicability at both mesoscales and microscales.

These articles provide a limited view of how algorithm development is substantially impacting our understanding of materials behavior and properties, and it enables us to create predictive models that can aid in science, engineering, and technology development at all length and time scales. The need for continued advancement in this field and the benefit of this progress are both obvious, as these efforts continue to better define the processingstructure-properties-performance pyramid known to all materials scientists and engineers.

\section{REFERENCES}

1. J.D. Honeycut and H.C. Andersen, J. Phys. Chem. 91, 4950 (1987).

2. C.L. Kelchner, S.J. Plimpton, and J.C. Hamilton, Phys. Rev. B 58, 11085 (1998).

3. A. Stukowski and K. Albe, Model. Simul. Mater. Sci. Eng. 18, 085001 (2010).

4. S. Torquato, Annu. Rev. Mater. Res. 32, 77 (2002).

5. Y. Jiao, F.H. Stillinger, and S. Torquato, Phys. Rev. E 76, 031110 (2007).

6. Y. Jiao, F.H. Stillinger, and S. Torquato, Phys. Rev. E 77, 031135 (2008). 